\title{
Chaos, control, and synchronization in some fractional-order difference equations
}

\author{
Amina-Aicha Khennaoui ${ }^{1}$, Adel Ouannas ${ }^{2}$, Samir Bendoukha ${ }^{3}$, Giuseppe Grassi ${ }^{4}$, Xiong Wang ${ }^{5}$, \\ Viet-Thanh Pham ${ }^{6 *}$ and Fawaz E. Alsaadi ${ }^{7}$
}

\author{
"Correspondence: \\ phamvietthanh@tdtu.edu.vn \\ ${ }^{6}$ Nonlinear Systems and \\ Applications, Faculty of Electrical \\ and Electronics Engineering, Ton \\ Duc Thang University, Ho Chi Minh \\ City, Vietnam \\ Full list of author information is \\ available at the end of the article
}

\begin{abstract}
In this paper, we propose three fractional chaotic maps based on the well known 3D Stefanski, Rössler, and Wang maps. The dynamics of the proposed fractional maps are investigated experimentally by means of phase portraits, bifurcation diagrams, and Lyapunov exponents. In addition, three control laws are introduced for these fractional maps and the convergence of the controlled states towards zero is guaranteed by means of the stability theory of linear fractional discrete systems. Furthermore, a combined synchronization scheme is introduced whereby the fractional Rössler map is considered as a drive system with the response system being a combination of the remaining two maps. Numerical results are presented throughout the paper to illustrate the findings.
\end{abstract}

MSC: 34A08; 34D06; 34H10

Keywords: Fractional discrete-time calculus; Fractional Stefanski map; Fractional Rössler map; Fractional Wang map; Control; Synchronization

\section{Introduction}

Chaotic discrete-time systems (maps) have received considerable attention over the last two decades due to their many applications in secure communications [1-4] and control [5]. Numerous maps have been proposed throughout the years including Hénon map [6], Lozi system [7], generalized Hénon map [8], Baier-Klein system [9], Stefanski map [10], Rössler map [11], and Wang map [12]. These maps exhibit a chaotic behavior in the sense that their trajectories are highly dependent on the system's initial conditions. Very recently, interest has grown from the research community in the study and applications of fractional discrete calculus. Fractional discrete systems have a major advantage over their conventional counterparts due to the infinite memory they feature, which allows for more flexibility in modeling and leads to a higher degree of chaotic behavior. In addition, fractional maps usually exhibit a chaotic attractor over a range of fractional orders, which increases their applicability in secure communications. Several studies have attempted to develop a complete framework for discrete fractional calculus and generalize the stability theory of conventional discrete calculus to the fractional domain [13-17]. However, since the topic of fractional discrete calculus is still new, to the best of our knowledge, very few fractional order chaotic maps have been proposed in the literature such as [18-21].

(c) The Author(s) 2019. This article is distributed under the terms of the Creative Commons Attribution 4.0 International License (http://creativecommons.org/licenses/by/4.0/), which permits unrestricted use, distribution, and reproduction in any medium, provided you give appropriate credit to the original author(s) and the source, provide a link to the Creative Commons license, and indicate if changes were made. 
When talking about chaotic systems in general, two of the main concerns are their control and synchronization. Control refers to the adaptive control of a given chaotic system with the aim of forcing its states to be asymptotically stable, usually converging towards zero [22, 23]. One of the applications of this topic is in robotics where the control of the chaotic motion of a rigid body is considered. No studies can be found in the literature regarding the control of fractional chaotic maps. The second major aspect of chaotic systems is their synchronization. In the revolutionary work of Pecora and Carroll [24], the authors showed that two Lorenz systems with different initial conditions can be controlled to follow the exact same trajectory. This was the seed that started the long use of chaotic systems in the field of communications. Throughout the years, many studies have considered the synchronization of integer-order chaotic and hyperchaotic maps including [25-29] but very few can be found for those of fractional-order [3034].

In this paper, we propose three fractional chaotic maps based on the Stefanski, Rössler, and Wang maps and study the existence of chaos and its control and synchronization. The following section reviews some important theory related to fractional discrete calculus, including the necessary notation and notes on the stability of linear fractional maps. Section 3 introduces the proposed fractional map based on the Stefanski discrete-time system, discusses its dynamics, and presents the related control scheme. Sections 4 and 5 present and investigate the dynamics and control of the fractional Rössler and Wang maps, respectively. Section 6 discusses the combined synchronization scheme and establishes the convergence of the synchronization errors, both analytically and numerically. Finally, Sect. 7 summarizes the results of the paper and poses ideas for future work.

\section{Fractional discrete-time calculus}

Before we start talking about chaotic fractional discrete-time systems and their control and synchronization, let us first recall some of the necessary theory related to the subject. Throughout our work, we will denote by ${ }^{C} \Delta_{a}^{v} X(t)$ the $v$-Caputo type delta difference of a function $X(t): \mathbb{N}_{a} \rightarrow \mathbb{R}$ with $\mathbb{N}_{a}=\{a, a+1, a+2, \ldots\}$ [14], which is of the form

$$
{ }^{C} \Delta_{a}^{v} X(t)=\Delta_{a}^{-(n-v)} \Delta^{n} X(t)=\frac{1}{\Gamma(n-v)} \sum_{s=a}^{t-(n-v)}(t-\sigma(s))^{(n-v-1)} \Delta^{n} X(s)
$$

for $v \notin \mathbb{N}$ being the fractional order, $t \in \mathbb{N}_{a+n-v}$, and $n=\lceil v\rceil+1$. In (1), the $v$ th fractional sum of $X(t)$ is defined similar to [13] as

$$
\Delta_{a}^{-v} X(t)=\frac{1}{\Gamma(v)} \sum_{s=a}^{t-v}(t-\sigma(s))^{(v-1)} X(s),
$$

with $v>0, \sigma(s)=s+1$. The term $t^{(v)}$ denotes the decreasing function defined in terms of the Gamma function $\Gamma$ as

$$
t^{(v)}=\frac{\Gamma(t+1)}{\Gamma(t+1-v)} .
$$


The following theorems provide the basis for the numerical analysis and stability theory that we will require later on when dealing with the proposed fractional-order discrete-time systems.

Theorem 1 ([21]) For the delta fractional difference equation

$$
\left\{\begin{array}{l}
{ }^{C} \Delta_{a}^{v} u(t)=f(t+v-1, u(t+v-1)) \\
\Delta^{k} u(t)=u_{k}, \quad n=\lceil v\rceil+1, k=0,1, \ldots, n-1
\end{array}\right.
$$

the equivalent discrete integral equation can be obtained as

$$
u(t)=u_{0}(t)+\frac{1}{\Gamma(v)} \sum_{s=a+n-v}^{t-v}(t-\sigma(s))^{(v-1)} f(s+v-1, u(s+v-1)), \quad t \in \mathbb{N}_{a+n},
$$

where

$$
u_{0}(t)=\sum_{k=0}^{m-1} \frac{(t-a)^{k}}{k} \Delta^{k} u(a)
$$

Theorem 2 ([35]) The zero equilibrium of the linear fractional-order discrete-time system

$$
{ }^{C} \Delta_{a}^{v} X(t)=\mathbf{M} X(t+v-1)
$$

where $X(t)=\left(x_{1}(t), \ldots, x_{n}(t)\right)^{T}, 0<v \leq 1, \mathbf{M} \in \mathbb{R}^{n \times n}$ and $\forall t \in \mathbb{N}_{a+1-v}$, is asymptotically stable if

$$
\lambda \in\left\{z \in \mathbb{C}:|z|<\left(2 \cos \frac{|\arg z|-\pi}{2-v}\right)^{v} \text { and }|\arg z|>\frac{v \pi}{2}\right\}
$$

for all the eigenvalues $\lambda$ of $\mathbf{M}$.

\section{Fractional-order Stefanski map}

\subsection{System model and dynamics}

In [10], Stefanski introduced a generalization of the standard Hénon map into 3dimensional space. The system is of the form

$$
\left\{\begin{array}{l}
x(n+1)=1+z(n)-\alpha y^{2}(n), \\
y(n+1)=1+\beta y(n)-\alpha x^{2}(n) \\
z(n+1)=\beta x(n)
\end{array}\right.
$$

where $x(n), y(n)$, and $z(n)$ are the states, $\alpha>0$, and $0<\beta<1$. The system has been studied extensively in the literature and is known to exhibit a hyperchaotic behavior for the bifurcation parameters $\beta=0.2$, and $\alpha \in[1.22,1.40]$. The resulting attractor for $\alpha=1.4$ and $\beta=0.2$ is depicted in Fig. 1 . 

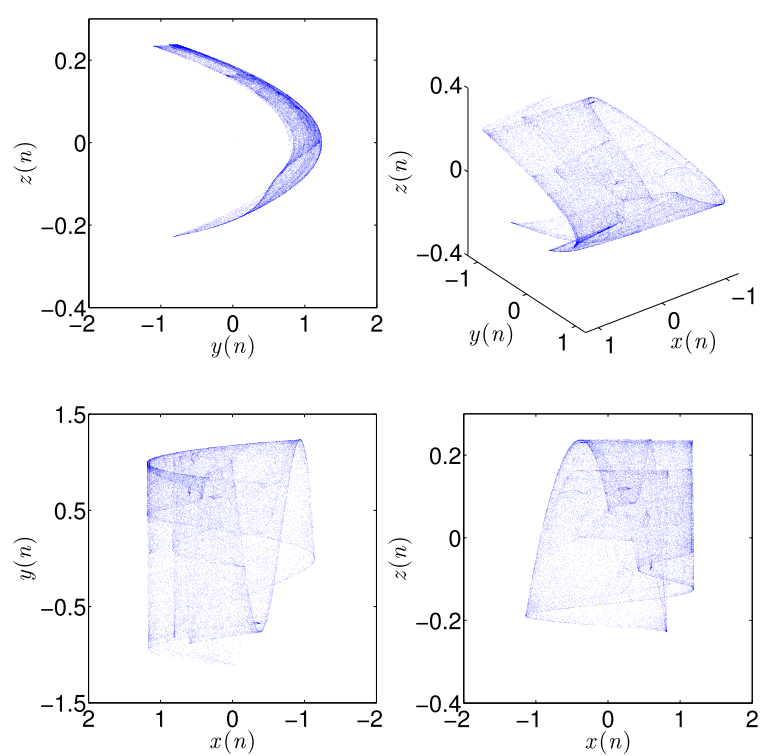

Figure 1 Phase portraits of the classical Stefanski attractor

Taking the first order difference of (9) yields

$$
\left\{\begin{array}{l}
\Delta x(n)=1+z(n)-\alpha y^{2}(n)-x(n), \\
\Delta y(n)=1+(\beta-1) y(n)-\alpha x^{2}(n), \\
\Delta z(n)=\beta x(n)-z(n) .
\end{array}\right.
$$

The $v$-Caputo type delta differences (1) can, therefore, be formulated for time $t \in \mathbb{N}_{a+1-v}$ and fractional order $0<v \leq 1$ as

$$
\left\{\begin{array}{l}
{ }^{C} \Delta_{a}^{v} x(t)=1+z(t-1+v)-\alpha y^{2}(t-1+v)-x(t-1+v), \\
{ }^{C} \Delta_{a}^{v} y(t)=1+(\beta-1) y(t-1+v)-\alpha x^{2}(t-1+v), \\
{ }^{C} \Delta_{a}^{v} z(t)=\beta x(t-1+v)-z(t-1+v) .
\end{array}\right.
$$

We will call system (11) the fractional-order Stefanski map. Suppose that $a=0$. Using Theorem 1, the numerical formulas for (11) become

$$
\left\{\begin{aligned}
x(t)= & x(0)+\frac{1}{\Gamma(v)} \sum_{s=a+1-v}^{t-v}(t-\sigma(s))^{(v-1)} \\
& \times\left(1+z(s-1+v)-\alpha y^{2}(s-1+v)-x(s-1+v)\right), \\
y(t)= & y(0)+\frac{1}{\Gamma(v)} \sum_{s=a+1-v}^{t-v}(t-\sigma(s))^{(v-1)} \\
& \times\left(1+\beta y(s-1+v)-\alpha x^{2}(s-1+v)-y(s-1+v)\right), \\
z(t)= & z(0)+\frac{1}{\Gamma(v)} \sum_{s=a+1-v}^{t-v}(t-\sigma(s))^{(v-1)} \\
& \times(\beta x(s-1+v)-z(s-1+v)),
\end{aligned}\right.
$$


with $\frac{(t-\sigma(s))^{(v-1)}}{\Gamma(v)}$ being a certain discrete kernel function. From (3), we obtain the kernel

$$
(t-\sigma(s))^{(v-1)}=\frac{\Gamma(t-s)}{\Gamma(t-s-v+1)} .
$$

Replacing $t-v$ by $n$ and $s+v$ by $j,(12)$ and (13) yield

$$
\left\{\begin{array}{l}
x(n)=x(0)+\frac{1}{\Gamma(v)} \sum_{j=1}^{n} \frac{\Gamma(n-j+v)}{\Gamma(n-j+1)}\left(1+z(j-1)-\alpha y^{2}(j-1)-x(j-1)\right), \\
y(n)=y(0)+\frac{1}{\Gamma(v)} \sum_{j=1}^{n} \frac{\Gamma(n-j+v)}{\Gamma(n-j+1)}(1+(\beta-1) y(j-1)-\alpha x(j-1)), \\
z(n)=z(0)+\frac{1}{\Gamma(v)} \sum_{j=1}^{n} \frac{\Gamma(n-j+v)}{\Gamma(n-j+1)}(\beta x(j-1)-z(j-1)),
\end{array}\right.
$$

where $x(0), y(0)$, and $z(0)$ are the initial conditions.

Before we go ahead and present control and synchronization schemes for the fractional map (11), let us first examine some important dynamics. Let $a=0$ and $x(0)=y(0)=$ $z(0)=0$. The phase portraits are displayed in Figs. 2 and 3 for different fractional orders. For the numerical simulation, we choose the step size $\Delta \alpha=0.001$. The bifurcation diagrams are plotted in Fig. 4 for different values of fractional order $v$. When $v=0.97$, the bifurcation diagrams show a period-doubling cascade route to chaos in the range $\alpha \in[1.1,1.4]$. As the value of $v$ decreases, the bifurcation diagram of the fractional order map (11) expands along the $\alpha$ axis and gradually shift to the left.

In addition to visualizing the effect of parameter $\alpha$ on the dynamics of the map, we have seen that the value of the fractional order $v$ has an impact on the dynamics. This has been further investigated by plotting the bifurcation of the fractional Stefanski map (11) taking $v$ as the critical parameter. The resulting bifurcation diagram when $(\alpha, \beta)=$ $(1.4,0.2)$ and $(x(0), y(0) z(0))=(0,0,0)$ is depicted in Fig. 5 . We see that chaos is apparent for the interval $v \in[0.915,1]$. As soon as $v$ drops below 0.915 , the states diverge towards infinity.
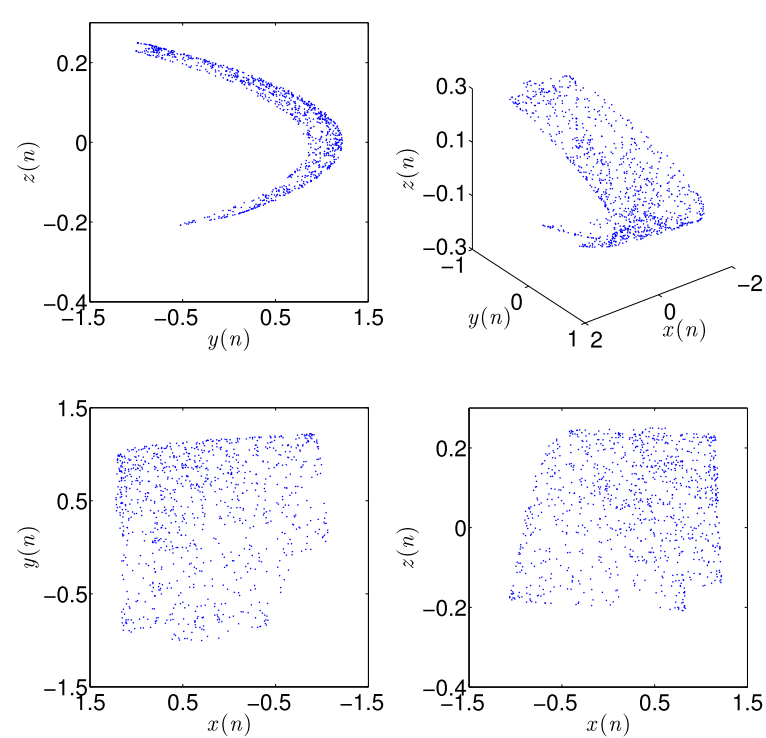

Figure 2 Phase portraits of the fractional-order Stefanski map with $v=0.97$ 

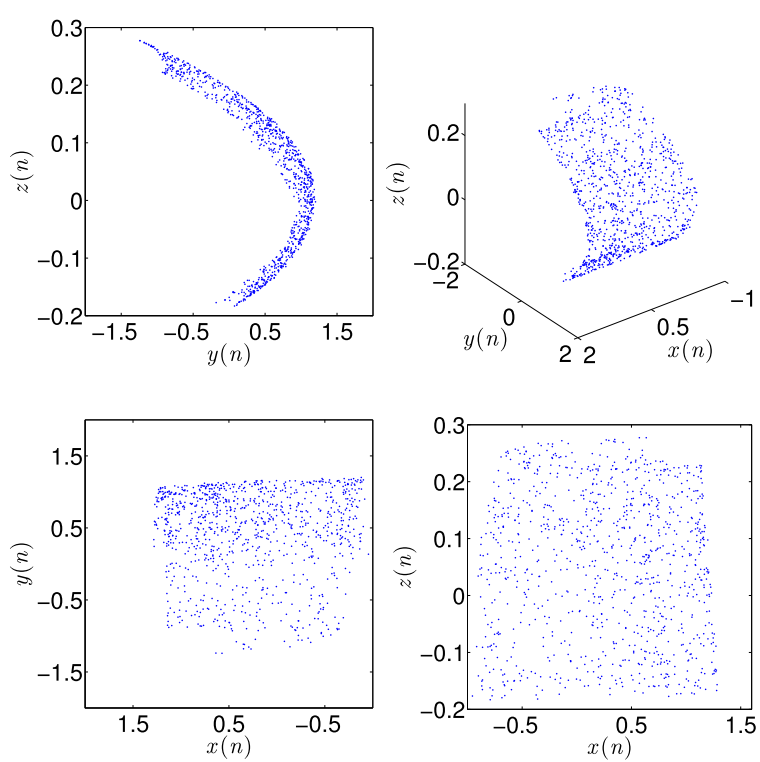

Figure 3 Phase space of the fractional-order Stefanski map for $v=0.969$
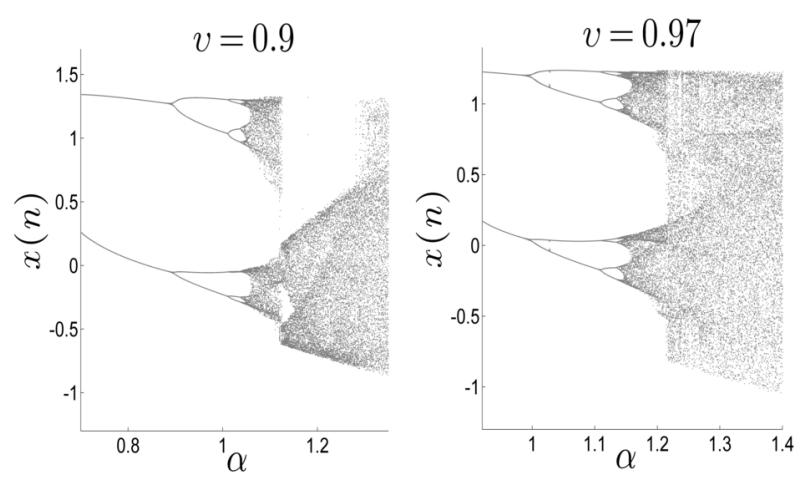

Figure 4 Bifurcation diagrams corresponding to the fractional Stefanski map with $\alpha$ as the critical parameter, $\beta=0.2$, and different fractional orders $v$

Although bifurcation plots clearly indicate the existence of chaos in the fractional map, it is usually more convenient to calculate or estimate the map's Lyapunov exponents. These are basically measures of the exponential divergence of two trajectories starting from infinitesimally close initial settings. A common method to estimate Lyapunov exponents for standard maps is by means of a QR decomposition of the time-varying Jacobian matrix. For fractional maps, the Jacobian matrix is subject to the same discrete memory effect as the map's states and thus can be calculated in a similar manner, see [36]. Figure 6 shows the estimated Lyapunov exponents for different values of the fractional order $v$. We see that the results agree with those of the bifurcation in the sense that lowering $v$ below 1 yields lower exponents to the point where they become negative, which coincides with a stable system. 

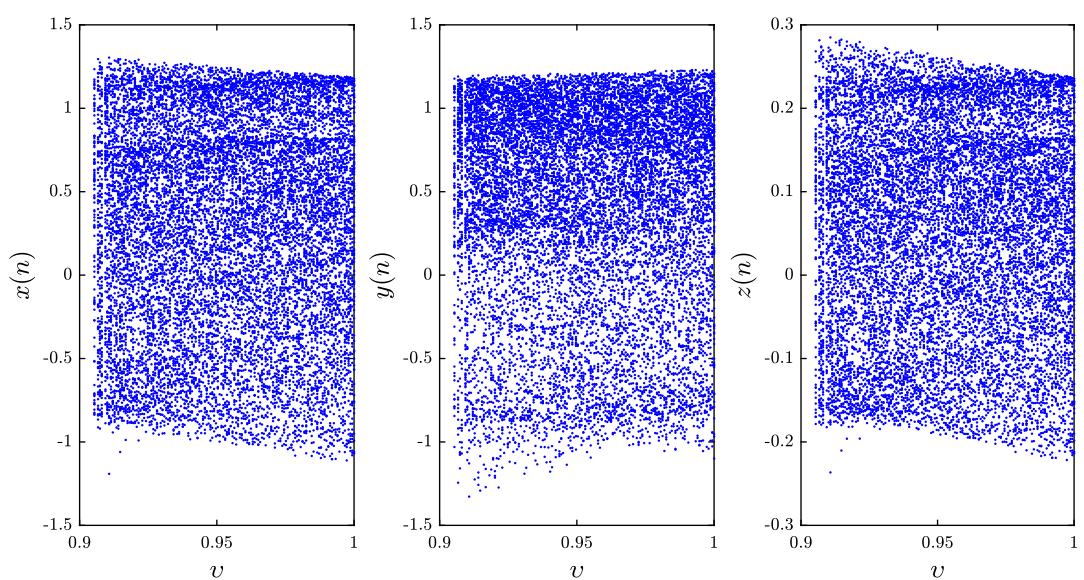

Figure 5 Bifurcation diagram of the fractional Stefanski map with $v \in[0,1]$ as the critical parameter, $(\alpha, \beta)=(1.4,0.2)$ and $(x(0), y(0) z(0))=(0,0,0)$
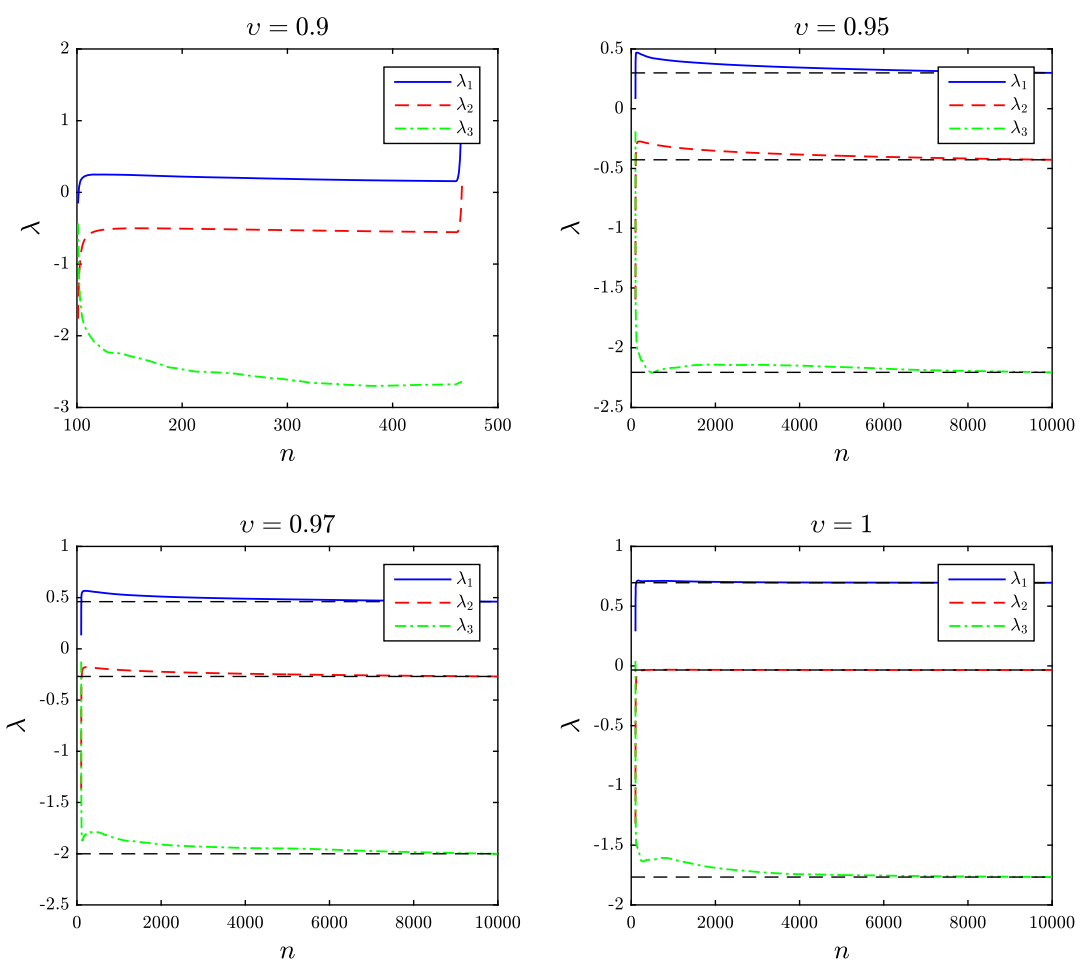

Figure 6 Estimated Lyapunov exponents of the fractional Stefanski map for $(\alpha, \beta)=(1.4,0.2)$, $(x(0), y(0) z(0))=(0,0,0)$, and different fractional orders

\subsection{Control laws}

When dealing with chaotic dynamical systems in general, particular interest is paid to our ability to control or stabilize these systems. By control, we refer to the addition of new adaptively updated terms to the chaotic system in order to force its states towards zero asymptotically. The following theorem proposes control laws for the fractional-order Stefanski map (11). 
Theorem 3 The 3D fractional-order Stefanski map (11) is stable under the 2D control law

$$
\left\{\begin{array}{l}
u_{x}(t)=\alpha y^{2}(t)-z(t)-1 \\
u_{y}(t)=\alpha x^{2}(t)-1
\end{array}\right.
$$

Proof Adding two control terms $u_{x}(t)$ and $u_{y}(t)$ to the fractional-order Stefanski map (11) results in the modified system

$$
\left\{\begin{aligned}
{ }^{C} \Delta_{a}^{v} x(t)= & 1+z(t-1+v)-\alpha y^{2}(t-1+v)-x(t-1+v) \\
& +u_{x}(t-1+v), \\
{ }^{C} \Delta_{a}^{v} y(t)= & 1+(\beta-1) y(t-1+v)-\alpha x^{2}(t-1+v) \\
& +u_{y}(t-1+v), \\
{ }^{C} \Delta_{a}^{v} z(t)= & \beta x(t-1+v)-z(t-1+v) .
\end{aligned}\right.
$$

Substituting the control law (15) yields the new dynamics

$$
\left\{\begin{array}{l}
{ }^{C} \Delta_{a}^{v} x(t)=-x(t-1+v), \\
{ }^{C} \Delta_{a}^{v} y(t)=(\beta-1) y(t-1+v), \\
{ }^{C} \Delta_{a}^{v} z(t)=\beta x(t-1+v)-z(t-1+v),
\end{array}\right.
$$

which can be described more compactly as

$$
{ }^{C} \Delta_{a}^{v}(x(t), y(t), z(t))^{T}=\mathbf{M}(x(t), y(t), z(t))^{T},
$$

with

$$
\mathbf{M}=\left(\begin{array}{ccc}
-1 & 0 & 0 \\
0 & \beta-1 & 0 \\
\beta & 0 & -1
\end{array}\right)
$$

We aim to show that the zero solution of (18) is globally asymptotically stable, which guarantees that all states converge towards zero at infinite time. In order to do so, we make use of the stability theory of linear fractional-order maps as described in Theorem 2. Simply, we can show that the eigenvalues of the matrix $\mathbf{M}$ are $\lambda_{1}=\lambda_{3}=-1$ and $\lambda_{2}=\beta-1$. It is easy to see that all the eigenvalues of the matrix $\mathbf{M}$ satisfy

$$
\left|\arg \lambda_{i}\right|=\pi>\frac{v \pi}{2}
$$

and

$$
\left|\lambda_{i}\right|<\left(2 \cos \frac{\left|\arg \lambda_{i}\right|-\pi}{2-v}\right)^{v}
$$

for $i=1,2,3$. According to Theorem 2 , the zero solution of (18) is globally asymptotically stable. Hence, the system is stabilized by means of control laws (15). 

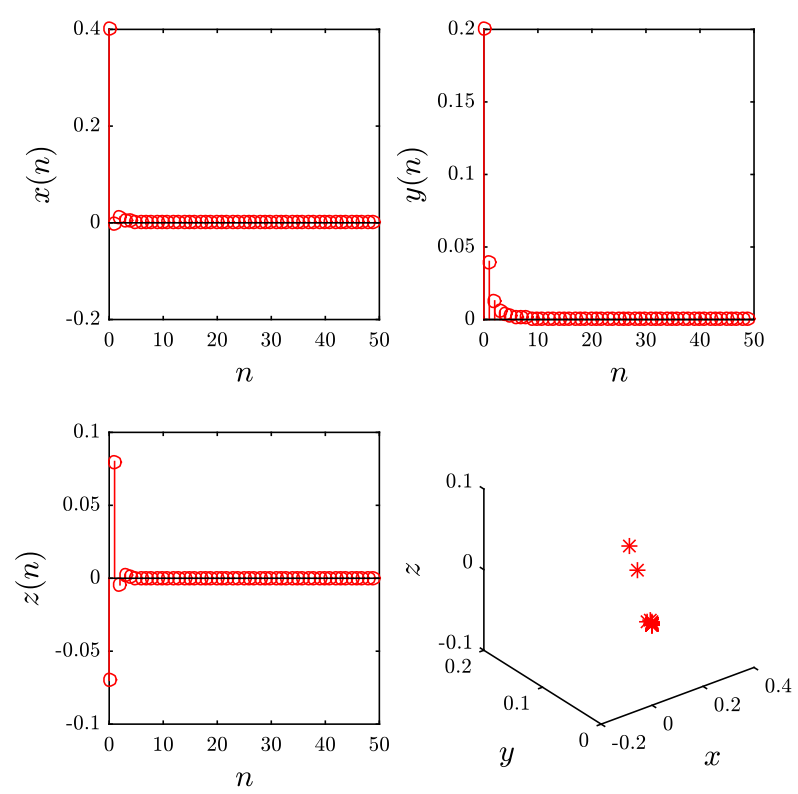

Figure 7 The time evolution of the controlled states for the fractional Stefanski map

In order to verify the result of Theorem 3, we implemented it numerically in Matlab. The discrete-time states are shown in Fig. 7. Clearly, the system is asymptotically stable and its states converge towards zero.

\section{Fractional-order discrete-time Rössler system}

\subsection{System model and dynamics}

The second map we are going to consider here is the 3D Rössler map introduced in [11] and given by

$$
\left\{\begin{array}{l}
x(n+1)=b_{1} x(n)(1-x(n))-b_{2}\left(z(n)+b_{3}\right)(1-2 y(n)), \\
y(n+1)=b_{4} y(n)(1-y(n))+b_{5} z(n), \\
z(n+1)=b_{6}\left(1-b_{7} x(n)\right)\left[\left(z(n)+b_{3}\right)(1-2 y(n))-1\right],
\end{array}\right.
$$

with states $x(n), y(n)$, and $z(n)$, and parameters $b_{1}=3.8, b_{2}=0.05, b_{3}=0.35, b_{4}=3.78$, $b_{5}=0.2, b_{6}=0.1$, and $b_{7}=1.9$. The Rössler map is well known and has been examined and applied in countless studies found in the literature. The phase-space portraits of the Rössler map for initial conditions $(x(0), y(0), z(0))=(0.1,0.2,-0.5)$ are displayed in Fig. 8.

The fractional-order map corresponding to (20) may be obtained in a similar manner to the fractional Stefanski map. Using the fractional discrete calculus notation in Sect. 2, we 

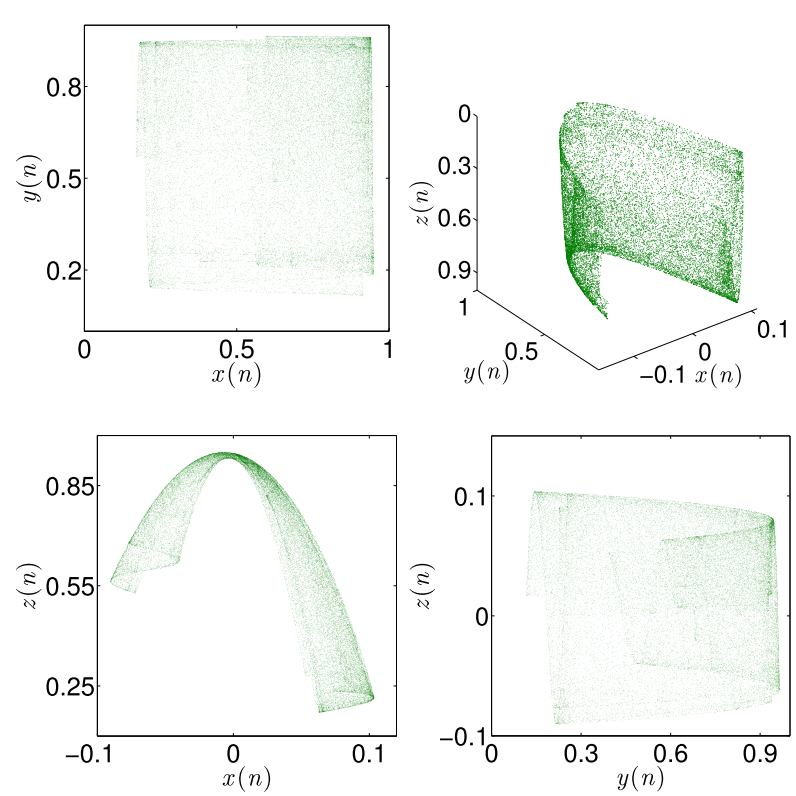

Figure 8 Phase space portraits of the standard Rössler map for $b_{1}=3.8, b_{2}=0.05, b_{3}=0.35, b_{4}=3.78$, $b_{5}=0.2, b_{6}=0.1$, and $b_{7}=1.9$

can write

$$
\left\{\begin{aligned}
{ }^{C} \Delta_{a}^{v} x(t)= & b_{1} x(t-1+v)(1-x(t-1+v)) \\
& -b_{2}\left(\left(z(t-1+v)+b_{3}\right)(1-2 y(t-1+v))\right)-x(t-1+v), \\
{ }^{C} \Delta_{a}^{v} y(t)= & b_{4} y(t-1+v)(1-y(t-1+v))+b_{5} z(t-1+v) \\
& -y(t-1+v), \\
{ }^{C} \Delta_{a}^{v} z(t)= & b_{6}\left(1-b_{7} x(t-1+v)\right) \\
& \times\left[\left(z(t-1+v)+b_{3}\right)(1-2 y(t-1+v))-1\right]-z(t-1+v),
\end{aligned}\right.
$$

for $t \in \mathbb{N}_{a-v+1}$, where $0<v \leq 1$. The corresponding numerical formulas follow from Theorem 1 and may be given by

$$
\left\{\begin{aligned}
x(n)= & x(a)+\frac{1}{\Gamma(v)} \sum_{j=1}^{n} \frac{\Gamma(n-j+v)}{\Gamma(n-j+1)} \\
& \times\left(b_{1} x(j-1)(1-x(j-1))-b_{2}\left(z(j-1)+b_{3}\right)(1-2 y(j-1))-x(j-1)\right), \\
y(n)= & y(a)+\frac{1}{\Gamma(v)} \sum_{j=1}^{n} \frac{\Gamma(n-j+v)}{\Gamma(n-j+1)} \\
& \times\left(b_{4} y(j-1)(1-y(j-1))+b_{5} z(j-1)-y(j-1)\right), \\
z(n)= & z(a)+\frac{1}{\Gamma(v)} \sum_{j=1}^{n} \frac{\Gamma(n-j+v)}{\Gamma(n-j+1)} \\
& \times\left(b_{6}\left(1-b_{7} x(j-1)\right)\left[\left(z(j-1)+b_{3}\right)(1-2 y(j-1))-1\right]-z(j-1)\right) .
\end{aligned}\right.
$$

In order to ensure that the fractional Rössler map (21) is chaotic, it helps to visualize its bifurcation plot. Let us consider a simple case where $a=0$ and $(x(0), y(0), z(0))=$ $(0.1,0.2,-0.5)$. Figures 9 and 10 depict the phase portraits for fractional orders $v=0.97$ and $v=0.91$, respectively. Through an experimental sweep of the fractional order, we found that the minimum value of $v$ for which the system exhibits a chaotic behavior is 

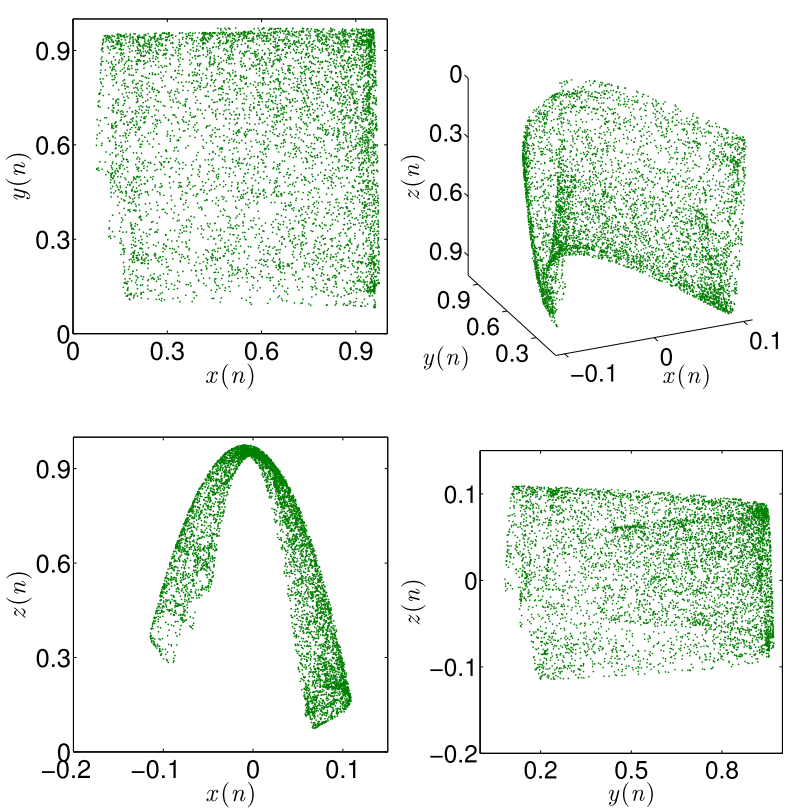

Figure 9 Phase portraits of the fractional order Rössler map for $v=0.97$
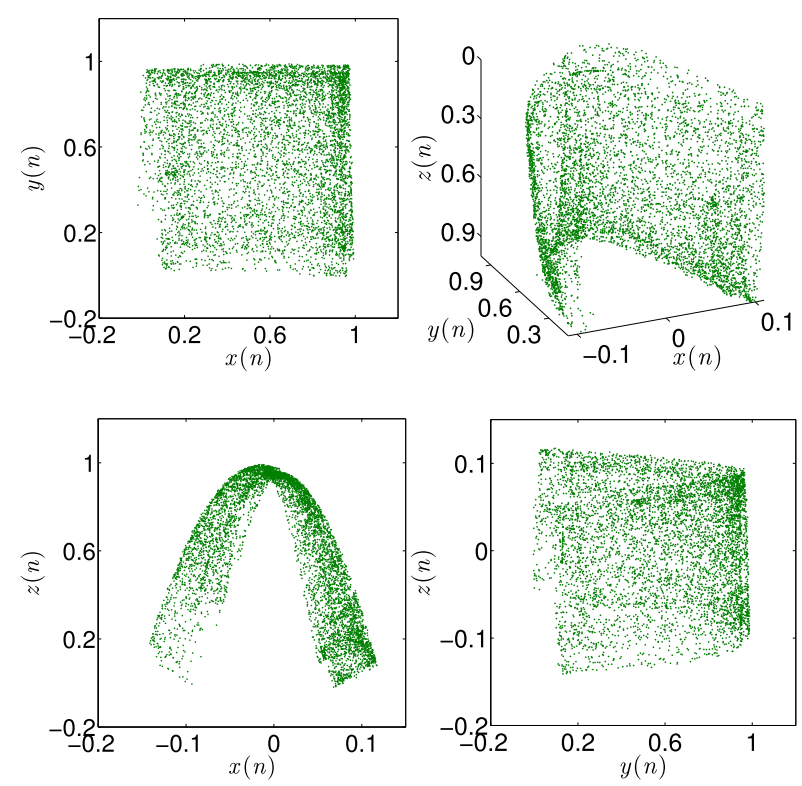

Figure 10 Phase portraits of the fractional order Rössler map for $v=0.91$

0.903. Figure 11 shows the bifurcation diagram for $v=0.97$ with $b_{1}$ as the critical parameter and $\left(b_{2}, b_{3}, b_{4}, b_{5}, b_{6}, b_{7}\right)=(0.05,0.35,3.78,0.2,0.1,1.9)$. The critical parameter was varied with the step size $\Delta b_{1}=0.001$. Figure 12 shows the bifurcation diagram of the fractional Rössler map with $v \in[0.9,1]$ as the critical parameter, $\left(b_{2}, b_{3}, b_{4}, b_{5}, b_{6}, b_{7}\right)=$ $(0.05,0.35,3.78,0.2,0.1,1.9)$ and $(x(0), y(0), z(0))=(0.1,0.2,-0.5)$. We see that chaos is only 
Figure 11 Bifurcation diagram of Rössler system with $a$ as the critical parameter and $v=0.97$
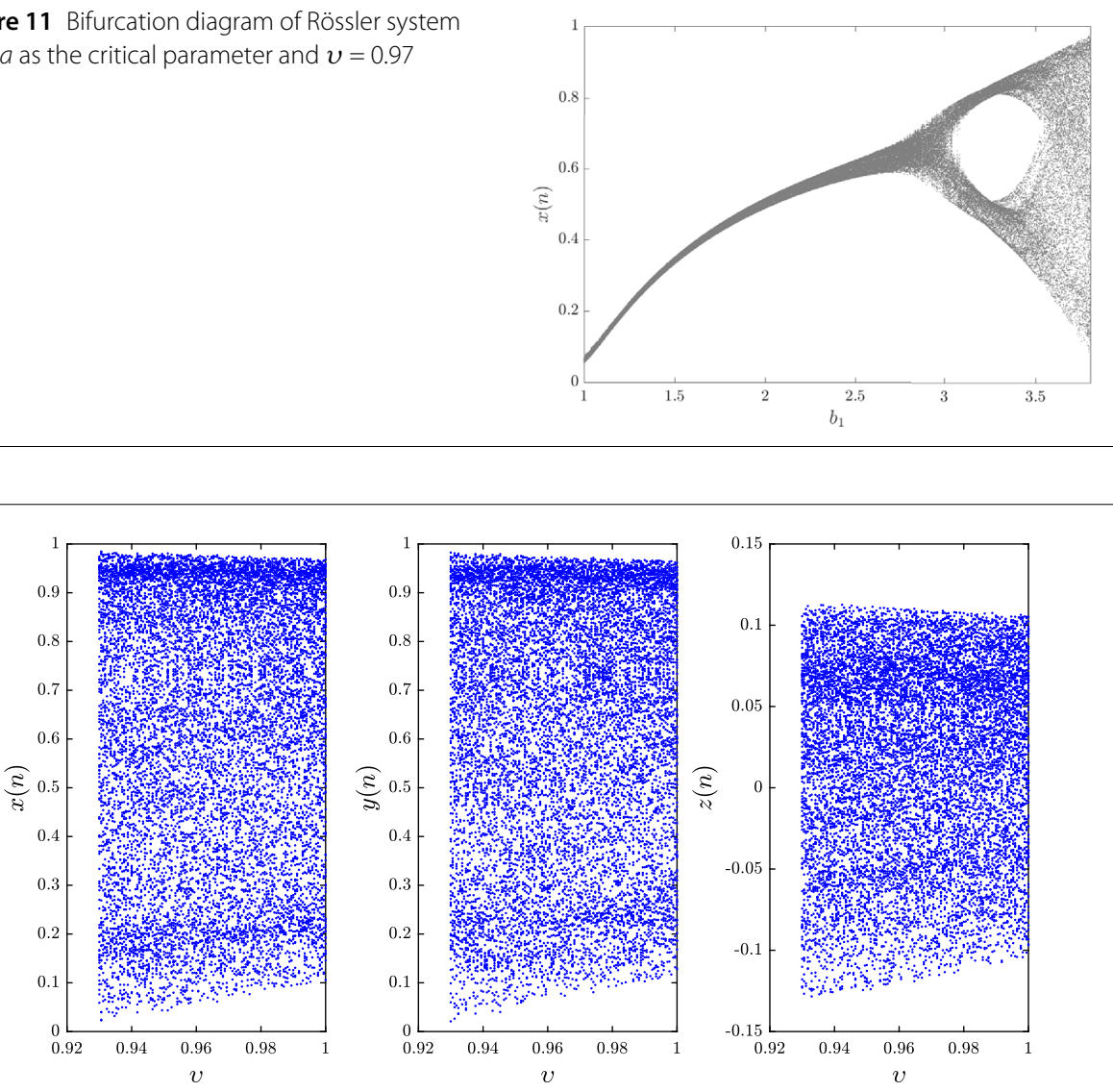

Figure 12 Bifurcation diagram of the fractional Rössler map with $v \in[0,1]$ as the critical parameter, $\left(b_{2}, b_{3}, b_{4}, b_{5}, b_{6}, b_{7}\right)=(0.05,0.35,3.78,0.2,0.1,1.9)$ and $(x(0), y(0), z(0))=(0.1,0.2,-0.5)$

observed for $v>v_{0} \approx 0.933$. Below $v_{0}$, the map becomes unstable and the states diverge towards infinity.

Using the same parameters and initial conditions, Fig. 13 shows the estimated Lyapunov exponents using the Jacobian matrix. For $v=1$, we observe that $\lambda_{1} \approx \lambda_{2}>0$, indicating a hyperchaotic nature of the fractional Rössler map. Similar to Stefanski map, as $v$ reduces, so do the Lyapunov exponents.

\subsection{Control laws}

In much the same way followed in the previous section, let us now propose adaptive laws to control the fractional Rössler map (21) and drive all of its states towards zero asymptotically.

Theorem 4 The fractional-order Rössler map becomes asymptotically stable subject to the control laws

$$
\left\{\begin{aligned}
u_{x}(t)= & -b_{1} x(t)+b_{1} x^{2}(t)-2 b_{2} z(t) y(t)+b_{2} b_{3}, \\
u_{y}(t)= & -b_{4} y(t)+b_{4} y^{2}(t), \\
u_{z}(t)= & 2 b_{6} b_{3} y(t)+2 b_{6} z(t) y(t)+b_{6}\left(1-b_{3}\right)+b_{6} b_{7} x(t) z(t) \\
& -2 b_{6} b_{7} x(t) y(t) z(t)-2 b_{3} b_{6} b_{7} x(t) y(t) .
\end{aligned}\right.
$$



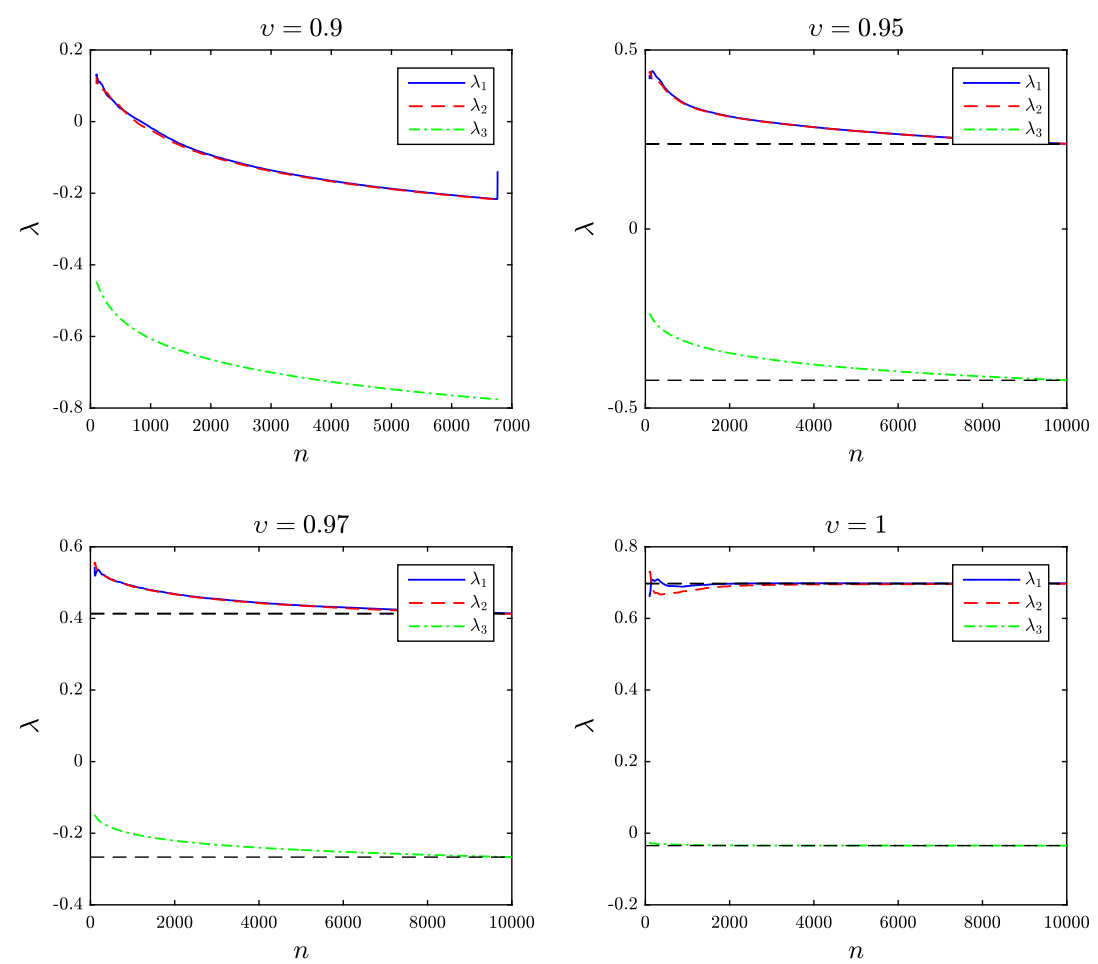

Figure 13 Estimated Lyapunov exponents of the fractional Rössler map for with

$\left(b_{2}, b_{3}, b_{4}, b_{5}, b_{6}, b_{7}\right)=(0.05,0.35,3.78,0.2,0.1,1.9),(x(0), y(0), z(0))=(0.1,0.2,-0.5)$, and different fractional orders $v$

Proof The controlled system corresponding to (21) is of the form

$$
\left\{\begin{aligned}
{ }^{C} \Delta_{a}^{v} x(t)= & b_{1} x(t-1+v)(1-x(t-1+v)) \\
& -b_{2}\left(\left(z(t-1+v)+b_{3}\right)(1-2 y(t-1+v))\right) \\
& -x(t-1+v)+u_{x}(t-1+v), \\
{ }^{C} \Delta_{a}^{v} y(t)= & b_{4} y(t-1+v)(1-y(t-1+v))+b_{5} z(t-1+v) \\
& -y(t-1+v)+u_{y}(t-1+v), \\
{ }^{C} \Delta_{a}^{v} z(t)= & b_{6}\left(1-b_{7} x(t-1+v)\right) \\
& \times\left[\left(z(t-1+v)+b_{3}\right)(1-2 y(t-1+v))-1\right] \\
& -z(t-1+v)+u_{z}(t-1+v) .
\end{aligned}\right.
$$

Substituting the control parameters stated in (23) yields the system dynamics

$$
{ }^{C} \Delta_{a}^{v}(x(t), y(t), z(t))^{T}=\mathbf{M}(x(t), y(t), z(t))^{T},
$$

where

$$
\mathbf{M}=\left(\begin{array}{ccc}
-1 & 2 b_{2} b_{3} & -b_{2} \\
0 & -1 & b_{5} \\
0 & 0 & b_{6}-1
\end{array}\right)
$$



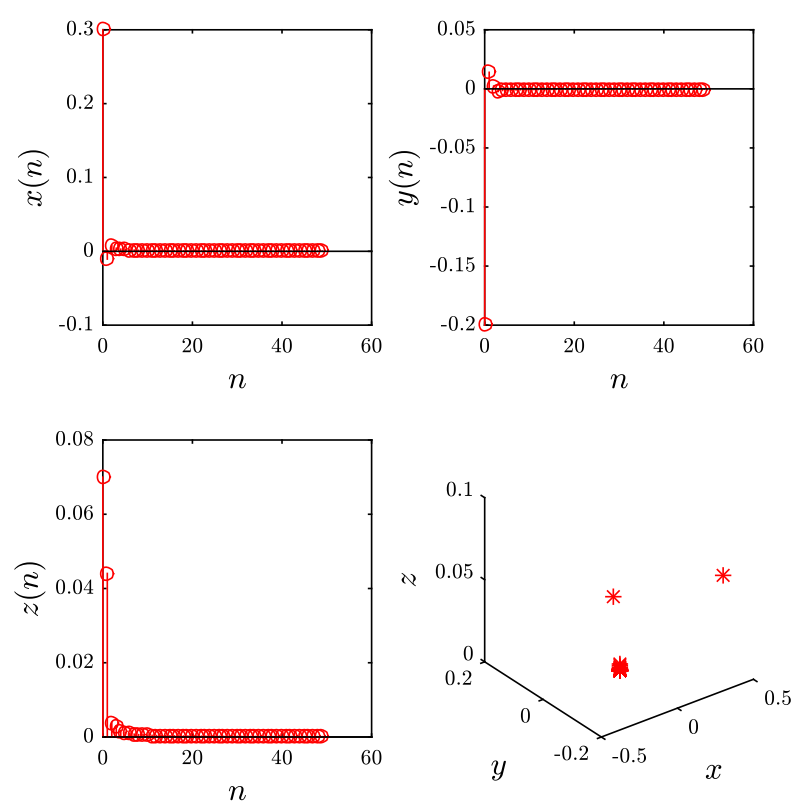

Figure 14 The time evolution of the controlled states for the fractional Rössler map

It is easy to see that the eigenvalues of $\mathbf{M}$ satisfy stability condition (8). By means of Theorem 2, we know that the zero solution is asymptotically stable and thus the system is stabilized.

Theorem 4 was put to the test using the same parameters and initial conditions stated at the beginning of this section and the control laws (23). Figure 14 shows the time evolution of the states, which clearly converge towards zero indicating successful stabilization.

\section{Fractional-order Wang map}

\subsection{System model and dynamics}

Another 3D chaotic map that has an interesting attractor is the hyperchaotic Wang map proposed in [12] and given by

$$
\left\{\begin{array}{l}
x(n+1)=a_{3} y(n)+\left(a_{4}+1\right) x(n), \\
y(n+1)=a_{1} x(n)+y(n)+a_{2} z(n), \\
z(n+1)=\left(a_{7}+1\right) z(n)+a_{6} y(n) z(n)+a_{5} .
\end{array}\right.
$$

Figure 15 shows the phase portraits for the following set of parameters:

$$
\left(a_{1}, a_{2}, a_{3}, a_{4}, a_{5}, a_{6}, a_{7}\right)=(-1.9,0.2,0.5,-2.3,2,-0.6,-1.9) .
$$

It is easy to see that the system exhibits chaos, a result that has been reported and studied in the literature. 

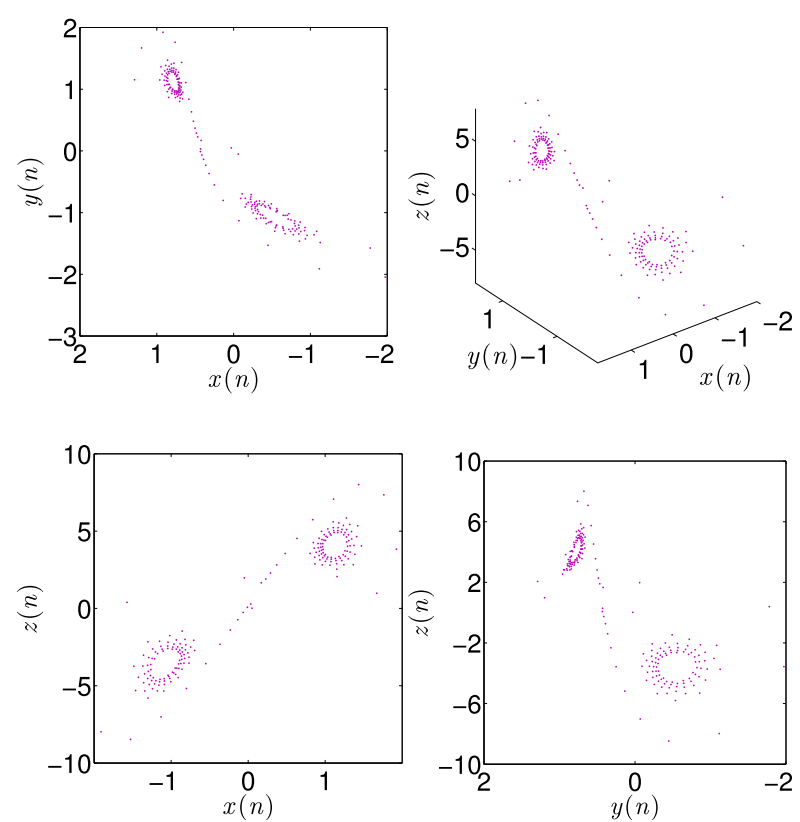

Figure 15 Phase portraits of the standard discrete-time Wang system

We follow the same lines of the previous two sections to arrive at the fractional-order discrete-time Wang map given by

$$
\left\{\begin{array}{l}
{ }^{C} \Delta_{a}^{v} x(t)=a_{3} y(t-1+v)+a_{4} x(t-1+v), \\
{ }^{C} \Delta_{a}^{v} y(t)=a_{1} x(t-1+v)+a_{2} z(t-1+v), \\
{ }^{C} \Delta_{a}^{v} z(t)=a_{7} z(t-1+v)+a_{6} y(t-1+v) z(t-1+v)+a_{5} .
\end{array}\right.
$$

The numerical formulas can be obtained in a similar fashion to the previous two sections by means of Theorem 1 . It can be easily shown that the fractional Wang map (28) is chaotic. Consider the case $a=0$ and initial conditions $(x(0), y(0), z(0))=(0.05,0.03,0.02)$. Figures 16 and 17 show the resulting attractors for the fractional orders $v=0.97$ and $v=0.969$, respectively. We have also plotted the bifurcation diagram with the critical parameter $a_{3}$ being varied at steps of $\Delta a_{.3}=0.001$ and the remaining parameters chosen as $\left(a_{1}, a_{2}, a_{4}, a_{5}, a_{6}, a_{7}\right)=(-1.9,0.2,-2.3,2,-0.6,-1.9)$. The bifurcation duration was set to $n=200$. The bifurcation diagrams are depicted in Fig. 18 for different fractional orders $v$. In Fig. 19, we show the bifurcation diagram of the fractional Wang map (28) with $v \in[0.9,1]$ as the critical parameter. We see that the map exhibits a chaotic behavior over a short interval of fractional orders. Chaos clearly disappears completely for $v<v_{0} \approx 0.915$. In fact, when $v<0.968$, the chaotic behavior is intermittent and has a very short range.

The Lyapunov exponents of the fractional Wang map (28) with the same previous parameters and initial conditions are depicted in Fig. 20. For $v=1$, we see that $\lambda_{1}>\lambda_{2}>0$, indicating hyperchaotic dynamics. This changes as the fractional order is made smaller. In fact, for $v=0.9,0>\lambda_{1}>\lambda_{2}>\lambda_{3}$, which leads to a stable map dynamic. 

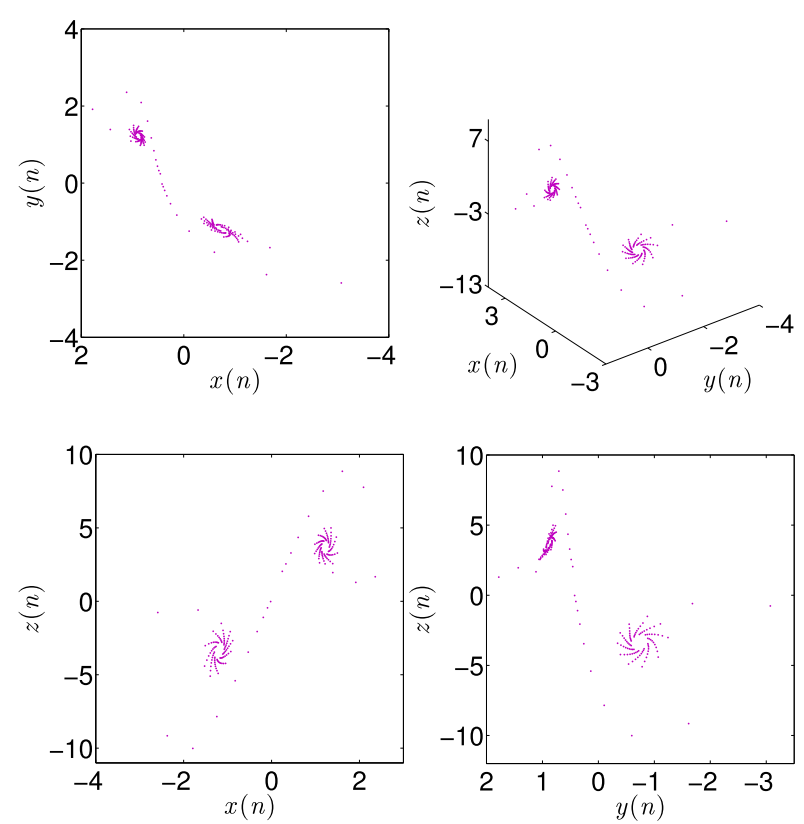

Figure 16 Phase space portraits of the fractional-order Wang map for $v=0.97$
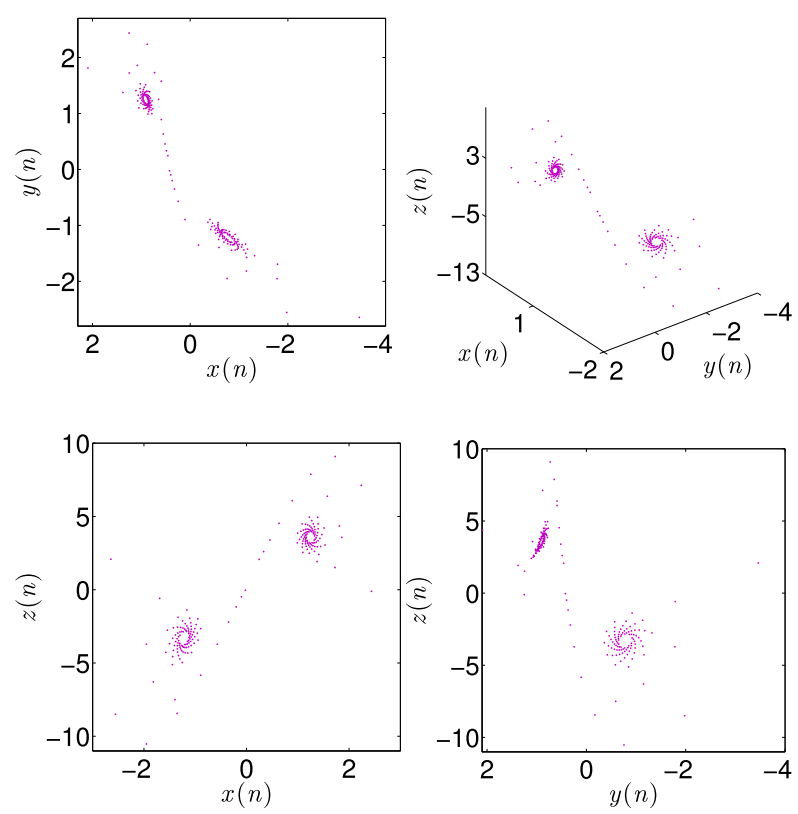

Figure 17 Phase space portraits of the fractional-order Wang map for $v=0.969$

\subsection{Control laws}

The following theorem presents the control laws to stabilize the fractional Wang map (28). The proof has been omitted as it follows the same lines of Theorems 3 and 4. It suffices to say that the eigenvalues of the resulting linear fractional error system is stable as a result of Theorem 2 for specific parameter values including the parameter set considered earlier in this section. 

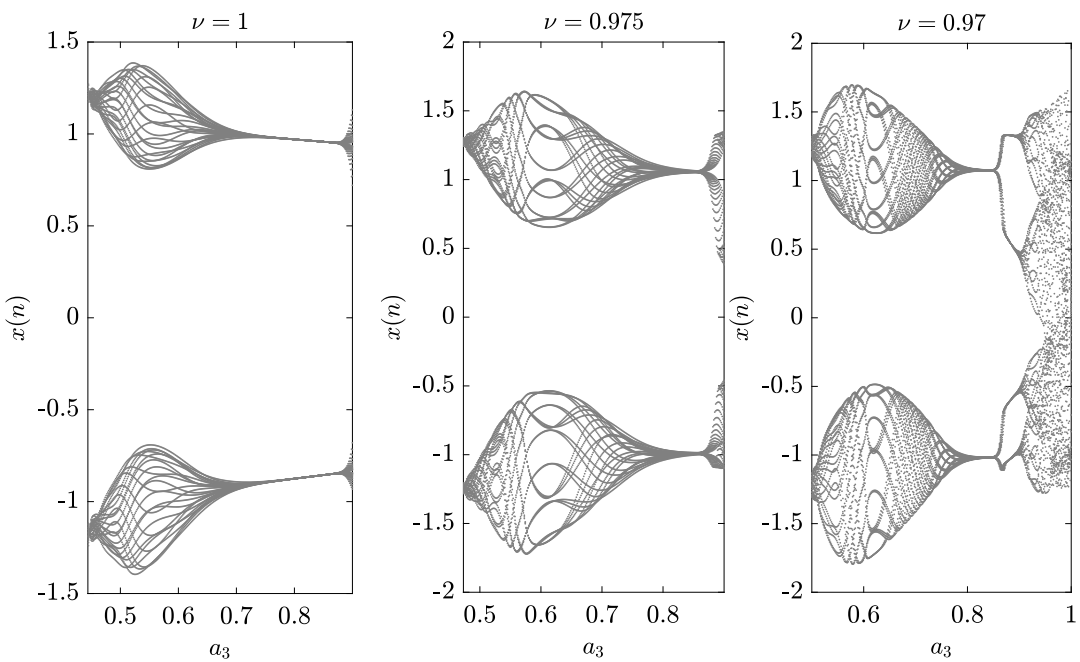

Figure 18 Bifurcation diagrams of the fractional-order Wang map for different fractional orders
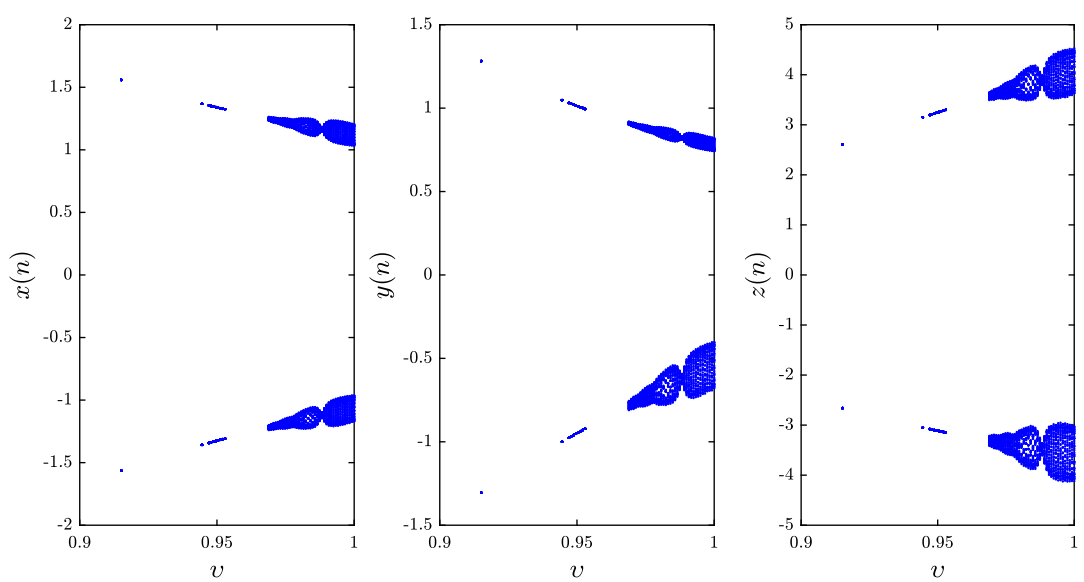

Figure 19 Bifurcation diagram of the fractional Rössler map with $v \in[0,1]$ as the critical parameter $\left(a_{1}, a_{2}, a_{4}, a_{5}, a_{6}, a_{7}\right)=(-1.9,0.2,-2.3,2,-0.6,-1.9)$ and $(x(0), y(0), z(0))=(0.05,0.03,0.02)$

Theorem 5 The fractional-order Wang map (28) is stabilized subject to the control laws

$$
\left\{\begin{array}{l}
u_{x}(t)=2 x(t), \\
u_{y}(t)=-a_{1} x(t)-y(t), \\
u_{z}(t)=z(t)-a_{6} y(t) z(t)-a_{5} .
\end{array}\right.
$$

Figure 21 depicts the time evolution of the states for the controlled fractional-order discrete-time Wang system. The states are observed to converge towards zero asymptotically, indicating that the system is stabilized.

\section{A combined synchronization scheme}

Although fractional-order discrete-time systems are still new and the literature related to them is limited, they have found applications in a number of fields, including secure 

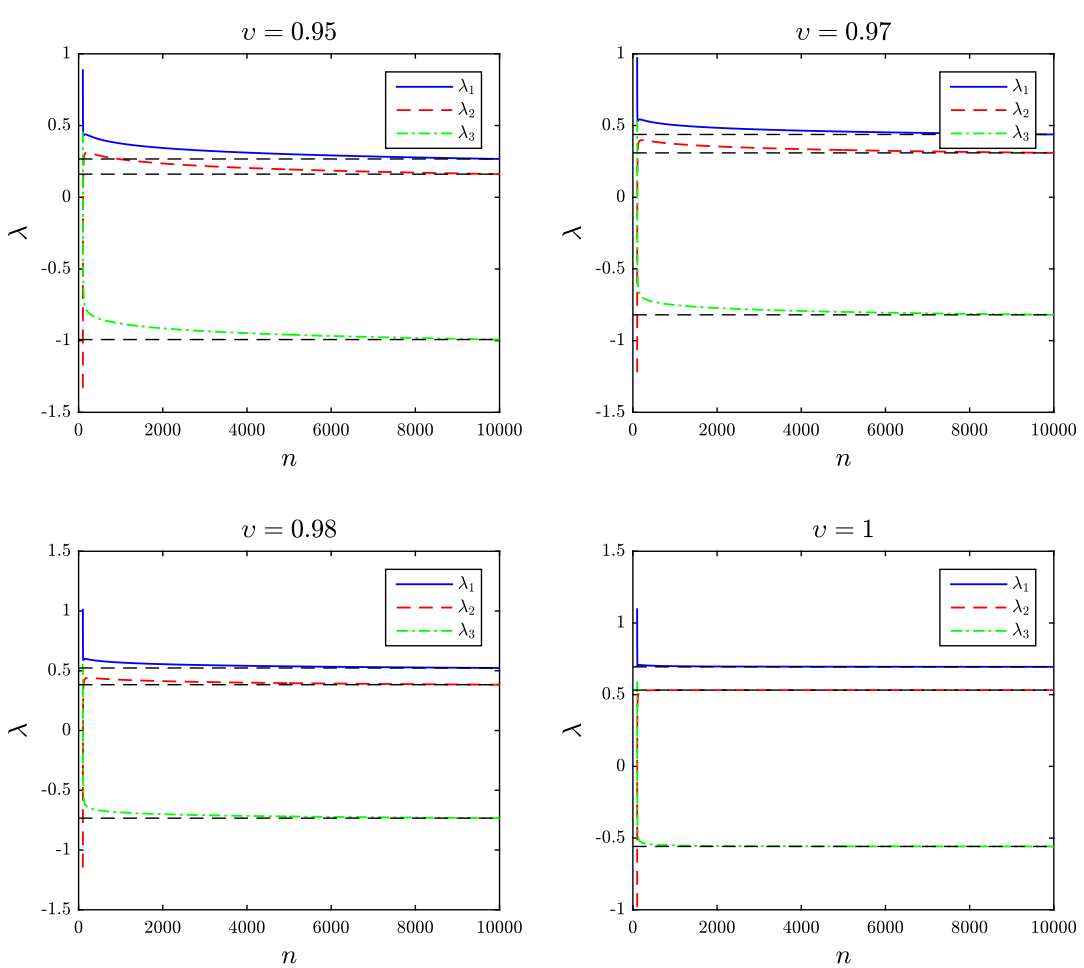

Figure 20 Estimated Lyapunov exponents of the fractional Wang map for with

$\left(a_{1}, a_{2}, a_{4}, a_{5}, a_{6}, a_{7}\right)=(-1.9,0.2,-2.3,2,-0.6,-1.9),(x(0), y(0), z(0))=(0.05,0.03,0.02)$, and different fractional orders $v$
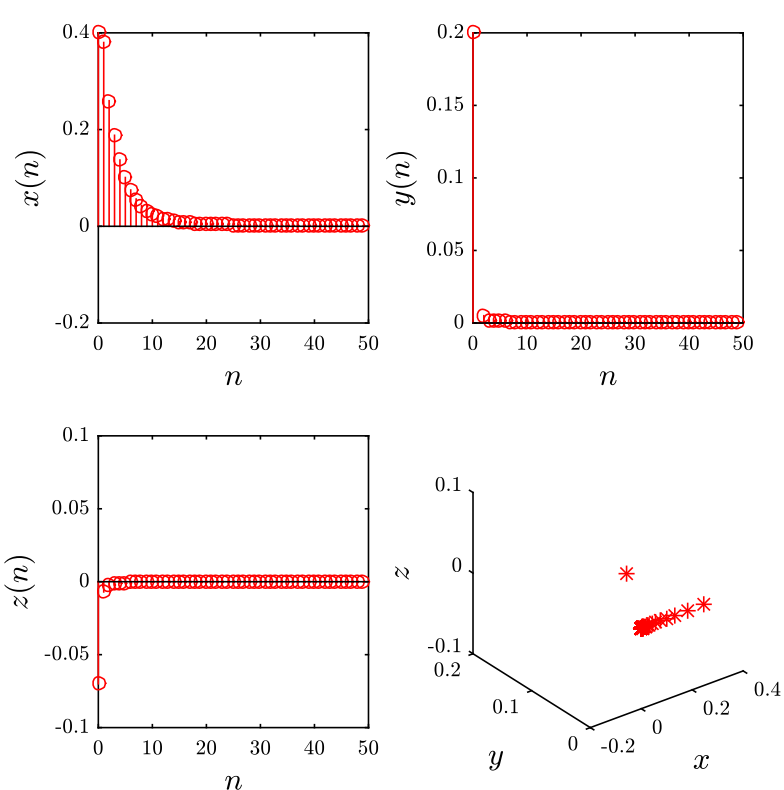

Figure $\mathbf{2 1}$ The time evolution of the controlled states for the fractional Wang map 
communications and data encryption as shown in [37], for instance. The application of this type of systems is mainly dependent on our ability to synchronize two systems starting from different initial conditions such that they end up following the same trajectory asymptotically. In this section, we propose a combined synchronization scheme for the three fractional maps discussed herein. We consider as our drive system the fractional Rössler map given for $t \in \mathbb{N}_{a+1-v}$ by

$$
\left\{\begin{aligned}
{ }^{C} \Delta_{a}^{v} x_{m}(t)= & b_{1} x_{m}(t-1+v)\left(1-x_{m}(t-1+v)\right) \\
& -b_{2}\left(z_{m}(t-1+v)+b_{3}\right)(1-2 y(t-1+v))-x_{m}(t-1+v), \\
{ }^{C} \Delta_{a}^{v} y_{m}(t)= & b_{4} y_{m}(t-1+v)\left(1-y_{m}(t-1+v)\right) \\
& +b_{5} z_{m}(t-1+v)-y_{m}(t-1+v), \\
{ }^{C} \Delta_{a}^{v} z_{m}(t)= & b_{6}\left(1-b_{7} x_{m}(t-1+v)\right) \\
& {\left[\left(z_{m}(t-1+v)+b_{3}\right)\left(1-2 y_{m}(t-1+v)\right)-1\right]-z_{m}(t-1+v) . }
\end{aligned}\right.
$$

As for the response system, we consider a combination of the fractional Stefanski map described by

$$
\left\{\begin{aligned}
{ }^{C} \Delta_{a}^{v} x_{s_{1}}(t)= & -x_{s_{1}}(t-1+v)+z_{s_{1}}(t-1+v)+1-\alpha y_{s_{1}}^{2}(t-1+v) \\
& +u_{1}(t-1+v), \\
{ }^{C} \Delta_{a}^{v} y_{s_{1}}(t)= & (\beta-1) y_{s_{1}}(t-1+v)+1-\alpha x_{s_{1}}^{2}(t-1+v)+u_{2}(t-1+v), \\
{ }^{C} \Delta_{a}^{v} z_{s_{1}}(t)= & \beta x_{s_{1}}(t-1+v)-z_{s_{1}}(t-1+v)+u_{3}(t-1+v),
\end{aligned}\right.
$$

and the fractional Wang map given by

$$
\left\{\begin{aligned}
{ }^{C} \Delta_{a}^{v} x_{s_{2}}(t)= & a_{3} y_{s_{2}}(t-1+v)+a_{4} x_{s_{2}}(t-1+v)+u_{4}(t-1+v), \\
{ }^{C} \Delta_{a}^{v} y_{s_{2}}(t)= & a_{1} x_{s_{2}}(t-1+v)+a_{2} z_{s_{2}}(t-1+v)+u_{5}(t-1+v), \\
{ }^{C} \Delta_{a}^{v} z_{s_{2}}(t)= & a_{7} z_{s_{2}}(t-1+v)+a_{6} y_{s_{2}}(t-1+v) z_{s_{2}}(t-1+v)+a_{5} \\
& +u_{6}(t-1+v),
\end{aligned}\right.
$$

where $t \in \mathbb{N}_{a+1-v}, u_{i}, i=1, \ldots, 6$, are control parameters to be designed, and

$$
\left\{\begin{array}{l}
(\alpha, \beta)=(1.4,0.2), \\
\left(a_{1}, a_{2}, a_{3}, a_{4}, a_{5}, a_{6}, a_{7}\right)=(-1.9,0.2,0.5,-2.3,2,-0.6,-1.9), \\
\left(b_{1}, b_{2}, b_{3}, b_{4}, b_{5}, b_{6}, b_{7}\right)=(3.8,0.05,0.35,3.78,0.2,0.1,1.9) .
\end{array}\right.
$$

The drive system (30) and the response systems (31)-(32) are said to be combinationsynchronized if there exist controllers $u_{1}(t), \ldots, u_{6}(t)$ such that the synchronization errors

$$
\left\{\begin{array}{l}
e_{1}(t)=x_{s_{1}}(t)+x_{s_{2}}(t)-x_{m}(t) \\
e_{2}(t)=y_{s_{1}}(t)+y_{s_{2}}(t)-y_{m}(t) \\
e_{3}(t)=z_{s_{1}}(t)+z_{s_{2}}(t)-z_{m}(t)
\end{array}\right.
$$


with $t \in \mathbb{N}_{a+1-v}$, converge to zero asymptotically, i.e.,

$$
\lim _{t \rightarrow \infty}\left|e_{i}(t)\right|=0, \quad i=1,2,3
$$

The following theorem proposes suitable adaptive laws for controllers $u_{1}(t), \ldots, u_{6}(t)$ in order to guarantee that (35) holds.

Theorem 6 Subject to

$$
\left\{\begin{array}{l}
u_{1}(t)=-y_{s_{1}}(t)+y_{m}(t)-z_{s_{1}}(t)-1+\alpha y_{s_{1}}^{2}(t)+b_{1} x_{m}(t)\left(1-x_{m}(t)\right), \\
u_{2}(t)=-z_{s_{1}}(t)-\beta y_{s_{1}}(t)-1+\alpha x_{s_{1}}^{2}(t)+b_{4} y_{m}(t)\left(1-y_{m}(t)\right), \\
u_{3}(t)=-\beta x_{s_{1}}(t)-a_{5}+b_{6}\left(1-b_{7} x_{m}(t)\right)\left[\left(z_{m}(t)+b_{3}\right)\left(1-2 y_{m}(t)\right)-1\right], \\
u_{4}(t)=-\left(a_{3}+1\right) y_{s_{2}}(t)-\left(a_{4}+1\right) x_{s_{2}}(t)-b_{2}\left(z_{m}(t)+b_{3}\right)\left(1-2 y_{m}(t)\right), \\
u_{5}(t)=-y_{s_{2}}(t)-a_{1} x_{s_{2}}(t)-\left(a_{2}+1\right) z_{s_{2}}(t)+\left(b_{5}+1\right) z_{m}(t), \\
u_{6}(t)=-z_{s_{2}}(t)-\left(a_{6} y_{s_{2}}(t)+a_{7}\right) z_{s_{2}}(t),
\end{array}\right.
$$

the drive system (30) and the response systems (31)-(32) are combination-synchronized.

Proof Taking the fractional differences of the synchronization errors (34) and substituting the proposed controllers (36) yields the error dynamics

$$
{ }^{C} \Delta_{a}^{v}\left(e_{1}(t), e_{2}(t), e_{3}(t)\right)^{T}=\mathbf{M}\left(e_{1}(t), e_{2}(t), e_{3}(t)\right)^{T}
$$

where

$$
\mathbf{M}=\left(\begin{array}{ccc}
-1 & -1 & 0 \\
0 & -1 & -1 \\
0 & 0 & -1
\end{array}\right) \text {. }
$$

In order to ensure that the drive and response systems are synchronized, we must establish that the errors in (37) converge towards zero asymptotically. It is easy to see that the matrix M satisfies stability condition (8) of Theorem 2. Hence, we find that the zero solution of the error system (37) is asymptotically stable and, consequently, the drive system (30) and response systems (31)-(32) are combination-synchronized.

Using the parameters specified in (33) with $a=0$ and the same initial conditions from previous sections, a Matlab program was implemented to track the time evolution of the errors (34) and ensure they converge to zero asymptotically. The results are depicted in Fig. 22. It is obvious that the combination-synchronization is successful. The errors clearly decay to zero and the sums of the slave states match those of the master.

\section{Concluding remarks and future work}

In this paper, we have considered the generalization of three well known 3D chaotic maps, namely the Stefanski, Rössler, and Wang maps, to fractional discrete calculus. The proposed fractional maps exhibit a chaotic behavior over a range of fractional orders as 

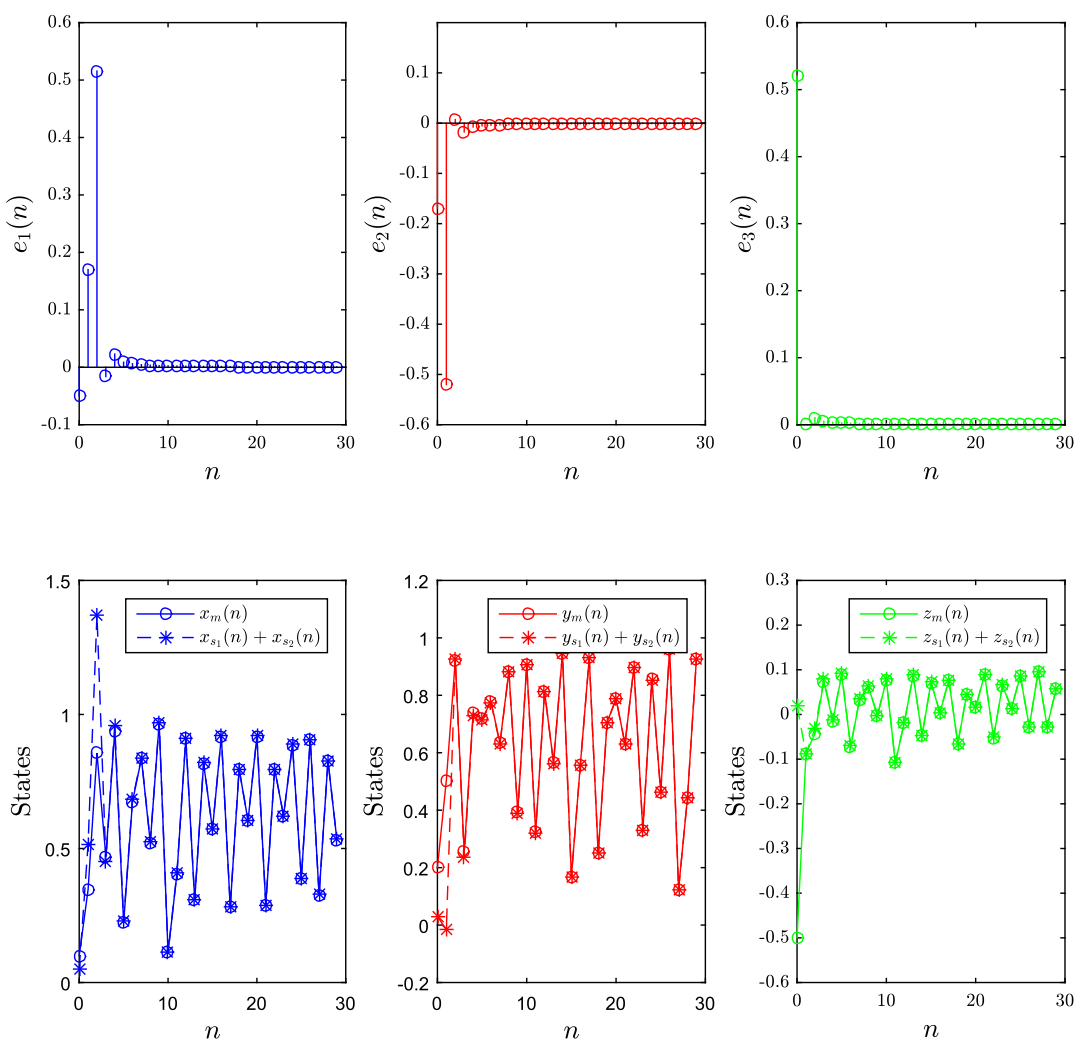

Figure 22 Time evolution of the synchronization errors for the proposed combined synchronization scheme

demonstrated by the phase portraits, as well as the bifurcation analysis and the estimation of Lyapunov exponents. The dynamics of these maps were analyzed by means of numerical methods. In addition, we presented three distinct stabilization laws for the proposed maps, whereby adaptive additive terms are included in the maps to drive their states towards zero asymptotically. The stability and convergence of these schemes was established by means of the stability theory of linear fractional discrete systems. Furthermore, we proposed a combination-synchronization scheme considering the fractional Rössler map as a drive system and a combination of the fractional Stefanski and Wang maps as the response system. The convergence of the stabilized states as well as the synchronization errors towards zero was illustrated by means of numerical simulation results.

It is well known that chaos is closely related to secure communications and data encryption. This is mainly attributed to the random-like nature of the chaotic states and trajectories. Throughout the last three decades a vast amount of literature has erupted in relation to the use of chaos in encryption. Numerous studies have demonstrated the feasibility of using chaotic maps in the generation of pseudo-random keys that may be used with conventional encryption schemes such as the data encryption standard (DES), the advanced encryption standard (AES), etc. Fractional chaotic maps come with the added dimension of the fractional order and thus lead to a higher degree of chaotic random-like behavior. In a future work, the proposed fractional maps will be utilized to encrypt data and images and demonstrate the importance of this kind of systems. 


\section{Acknowledgements}

The authors acknowledge Prof. GuanRong Chen, Department of Electronic Engineering, City University of Hong Kong for suggesting many helpful references.

\section{Funding}

Xiong Wang was supported by the National Natural Science Foundation of China (No. 61601306) and Shenzhen Overseas High Level Talent Peacock Project Fund (No. 20150215145C).

\section{Availability of data and materials}

Not applicable.

\section{Competing interests}

The authors declare that they have no competing interests.

\section{Authors' contributions}

AAK, AO and SB suggested the model, helped in results' interpretation and manuscript evaluation. GG and XW and VTP helped to evaluate, revise and edit the manuscript. GG, XW and FEA supervised the development of the work. VTP and FEA drafted the article. All authors read and approved the final manuscript.

\section{Author details}

'Laboratory of Dynamical System and Control, University of Larbi Ben M'hidi, Oum El Bouaghi, Algeria. ${ }^{2}$ Department of Mathematics and Computer Science, University of Larbi Tebessi, Tebessa, Algeria. ${ }^{3}$ Electrical Engineering Department, College of Engineering at Yanbu, Taibah University, Medina, Saudi Arabia. ${ }^{4}$ Dipartimento Ingegneria Innovazione, Universita del Salento, Lecce, Italy. ${ }^{5}$ Institute for Advanced Study, Shenzhen University, Shenzhen, P.R. China. ${ }^{6}$ Nonlinear Systems and Applications, Faculty of Electrical and Electronics Engineering, Ton Duc Thang University, Ho Chi Minh City, Vietnam. ${ }^{7}$ Department of Information Technology, Faculty of Computing and IT, King Abdulaziz University, Jeddah, Saudi Arabia.

\section{Publisher's Note}

Springer Nature remains neutral with regard to jurisdictional claims in published maps and institutional affiliations.

Received: 18 October 2018 Accepted: 16 September 2019 Published online: 26 September 2019

\section{References}

1. Lian, K.Y., Chiang, T.S., Liu, P.: Discrete-time chaotic systems: applications in secure communications. Int. J. Bifurc Chaos 10, $2193(2000)$

2. Feki, M., Robert, B., Gelle, G., Colas, M.: Secure digital communication using discrete-time chaos synchronization. Chaos Solitons Fractals 18, 881-890 (2003)

3. Guo, L.J., Geng, X.Y.: Chaos communication based on synchronization of discrete-time chaotic systems. Chin. Phys. $14,274(2005)$

4. Stork, M.: Digital chaotic systems examples and application for data transmission. In: Proc. Int. Conf. Electrical \& Electronics Eng. (ELECO'2009), Bursa, Turkey, pp. 78-82 (2009)

5. Kocarev, L., Szczepanski, J., Amigo, J.M., Tomovski, l.: Discrete chaos-I: theory. IEEE Trans. Circuits Syst. I, Regul. Pap. 53, 1300-1309 (2006)

6. Hénon, M.: A two-dimensional mapping with a strange attractor. Commun. Math. Phys. 50, 69-77 (1976)

7. Lozi, R.: Un atracteur étrange du type attracteur de hénon. J. Phys. 39, 9-10 (1978)

8. Hitzl, D., Zele, F.: An exploration of the Hénon quadratic map. Phys. D, Nonlinear Phenom. 14, 305-326 (1985)

9. Baier, G., Sahle, S.: Design of hyperchaotic flows. Phys. Rev. E 51, 2712-2714 (1995)

10. Stefanski, K.: Modelling chaos and hyperchaos with 3D maps. Chaos Solitons Fractals 9, $83-93$ (1998)

11. Itoh, M., Yang, T., Chua, L.: Conditions for impulsive synchronization of chaotic and hyperchaotic systems. Int. J. Bifurc. Chaos 11, 551-558 (2001)

12. Wang, X.Y.: Chaos in Complex Nonlinear Systems. Publishing House of Electronics Industry, Beijing (2003)

13. Atici, F.M., Eloe, P.W.: Discrete fractional calculus with the nabla operator. Electron. J. Qual. Theory Differ. Equ. Spec. Ed. | 2009, 3, 1-12 (2009)

14. Abdeljawad, T.: On Riemann and Caputo fractional differences. Comput. Math. Appl. 62, 1602-1611 (2011)

15. Abdeljawad, T., Baleanu, D., Jarad, F., Agarwal, R.P.: Fractional sums and differences with binomial coefficients. Discrete Dyn. Nat. Soc. 2013, 104173 (2013)

16. Goodrich, C., Peterson, A.C.: Discrete Fractional Calculus. Springer, German (2015)

17. Baleanu, D., Wu, G., Bai, Y., Chen, F.: Stability analysis of Caputo-like discrete fractional systems. Commun. Nonlinear Sci. Numer. Simul. 48, 520-530 (2017)

18. Wu, G., Baleanu, D.: Discrete fractional logistic map and its chaos. Nonlinear Dyn. 75, 283-287 (2013)

19. Hu, T.: Discrete chaos in fractional Hénon map. Appl. Math. 5, 2243-2248 (2014)

20. Shukla, M.K., Sharma, B.B.: Investigation of chaos in fractional order generalized hyperchaotic Hénon map. Int. J. Electron. Commer. 78, 265-273 (2017)

21. Wu, G.C., Baleanu, D.: Discrete chaos in fractional delayed logistic maps. Nonlinear Dyn. 80, 1697-1703 (2015)

22. Boccaletti, S., Grebogi, C., Lai, Y.C., Mancini, H., Maza, D.: The control of chaos: theory and applications. Phys. Rep. 329, 103-197 (2000)

23. Fradkov, A.L., Evans, R.J., Andrievsky, B.R.: Control of chaos: methods and applications in mechanics. Philos. Trans. R. Soc. A, Math. Phys. Eng. Sci. 364, 2279-2307 (2006)

24. Pecora, L.M., Carrol, T.L.: Synchronization in chaotic systems. Phys. Rev. A 64, 821 (1990) 
25. Ouannas, A., Azar, A.T., Abu-Saris, R.: A new type of hybrid synchronization between arbitrary hyperchaotic maps. Int. J. Mach. Learn. Cybern. 8, 1887-1894 (2017)

26. Ouannas, A., Grassi, G.: A new approach to study co-existence of some synchronization types between chaotic maps with different dimensions. Nonlinear Dyn. 86, 1319-1328 (2016)

27. Ouannas, A., Odibat, Z:: Generalized synchronization of different dimensional chaotic dynamical systems in discrete-time. Nonlinear Dyn. 81, 765-771 (2015)

28. Ouannas, A.: A new generalized-type of synchronization for discrete chaotic dynamical system. J. Comput. Nonlinear Dyn. 10, 061019 (2015)

29. Grassi, G., Ouannas, A., Pham, V.T.: A general unified approach to chaos synchronization in continuous-time systems (with or without equilibrium points) as well as in discrete-time systems. Arch. Control Sci. 28, 135-154 (2018)

30. Wu, G., Baleanu, D.: Chaos synchronization of the discrete fractional logistic map. Signal Process. 102, 96-99 (2014)

31. Wu, G., Baleanu, D., Xie, H., Chen, F.: Chaos synchronization of fractional chaotic maps based on the stability condition. Physica A 460, 374-383 (2016)

32. Liu, Y.: Chaotic synchronization between linearly coupled discrete fractional Hénon maps. Indian J. Phys. 90, 313-317 (2016)

33. Megherbi, O., Hamiche, H., Djennoune, S., Bettayeb, M.: A new contribution for the impulsive synchronization of fractional-order discrete-time chaotic systems. Nonlinear Dyn. 90, 1519-1533 (2017)

34. Huang, L.L., Baleanu, D., Wu, G.C., Zeng, S.D.: A new application of the fractional logistic map. Rom. J. Phys. 61, $1172-1179(2016)$

35. Cermak, J., Gyori, I., Nechvatal, L.: On explicit stability condition for a linear fractional difference system. Fract. Calc. Appl. Anal. 18, 651-672 (2015)

36. Wu, G.C., Baleanu, D.: Jacobian matrix algorithm for Lyapunov exponents of the discrete fractional maps. Commun. Nonlinear Sci. Numer. Simul. 22, 95-100 (2015)

37. Kassim, S., Hamiche, H., Djennoune, S., Bettayeb, M.: A novel secure image transmission scheme based on synchronization of fractional-order discrete-time hyperchaotic systems. Nonlinear Dyn. 88, 2473-2489 (2017)

\section{Submit your manuscript to a SpringerOpen ${ }^{\circ}$ journal and benefit from:}

- Convenient online submission

- Rigorous peer review

- Open access: articles freely available online

- High visibility within the field

Retaining the copyright to your article

Submit your next manuscript at $\boldsymbol{\nabla}$ springeropen.com 